\title{
Interação Clínica entre Moléstia de Chagas e Hipertensão Arterial Primária em um Serviço de Referência Ambulatorial
}

\author{
Maria Elena Guariento, José Eduardo Bagnara O rosz, José A ntonio Rocha Gontijo \\ Campinas, SP
}

\begin{abstract}
Objetivo - Caracterizar a associação entre doença de Chagas e hipertensão arterial primária.

Métodos - Estudaram-se os chagásicos crônicos acompanhados pelo Grupo de Estudos em Doença de Chagas (Unicamp), no periodo de 15 anos, considerando-se: hipertensão arterial, forma clínica da doença, sexo, idade e raça. Confrontaram-se dados dos chagásicos hipertensos com os dos não hipertensos.

Resultados - Constatou-se que entre 878 chagásicos, $37 \%$ eram também hipertensos, dos quais $65 \%$ tinham cardiopatia, contra $49 \%$ do grupo normotenso; $31 \%$ dos chagásicos hipertensos apresentavam descompensação hemodinâmica, contra $24 \%$ dos não hipertensos. Entre os chagásicos hipertensos $41 \%$ eram negróides e $35 \%$ caucasóides; $50 \%$ dos chagásicos hipertensos tinham mais de 45 anos contra $29 \%$ dos não hipertensos.

Conclusão - A associação entre moléstia de Chagas e hipertensão arterial primária cursou com um freqüente e mais grave comprometimento cardíaco, sendo esta mais prevalente nas faixas etárias mais elevadas.
\end{abstract}

Palavras-chave: doença de Chagas, hipertensão arterial primária, cardiopatia

\section{Clinical Relationship between Chagas' Disease and Primary Arterial Hypertension in an Outpatient Reference Service}

Purpose - To evaluate the clinical relationship between Chagas' disease and primary arterial hypertension.

Methods - The study involved 878 chronic chagasic outpatients followed by the Chagas' Disease Study Group of the University Hospital (Unicamp), over a 15-year period. Initially, the age, gender, race, clinical form of Chagas' disease and the presence of hypertension were noted for each patient. Subsequently, the data for matched hypertensive and normotensive chagasic patients were compared.

Results - Of the chagasic patients $37 \%$ were hypertensive, of those, $65 \%$ had some form of heart disease compared to $49 \%$ in the normotensive group. Of those patients with Chagas' disease and arterial hypertension, $41 \%$ were blacks and $35 \%$ were caucasians. Fifty percent of the hypertensive chagasic patients were over 45 years old compared to only $29 \%$ of the nonhypertensive patients.

Conclusion - Most of the hypertensive chagasic patients were 45 or more years old and showed some degree of heart failure compared to the normotensive group.

Key-words: Chagas' disease, primary arterial hypertension, cardiopathy

Arq Bras Cardiol, volume 70 (nº 6), 431-434, 1998

Existem controvérsias na literatura quanto ao comportamento das cifras tensionais em pacientes chagásicos crônicos ${ }^{1,2}$. Entretanto, alguns autores têm relatado até mesmo

Faculdade de Ciências Médicas - UNICAMP - Campinas

Correspondência: Maria Elena Guariento - Depto de Clínica Médica - Cidade Universitária Zeferino Vaz - UNICAMP - Distrito de Barão Geraldo - 13083-970 - Campinas, SP

Recebido para publicação em 18/11/97

Aceito em 25/3/98 uma prevalência de níveis pressóricos mais elevados na população portadora de infecção pelo T.cruzi quando essa é confrontada com os não portadores desta afecção ${ }^{3-5}$.

No sentido de se caracterizar uma associação entre a presença de hipertensão arterial (HA) primária e doença de Chagas, bem como a repercussão clínica dessa associação, foi desenvolvido este estudo, utilizando-se os pacientes chagásicos crônicos acompanhados no Ambulatório do Grupo de Estudos em Doença de Chagas (GEDoCh)/HC DCM/FCM - Unicamp. 


\section{Métodos}

Foram avaliados 878 pacientes chagásicos com pelo menos duas reações sorológicas francamente positivas (reação de fixação de complemento $\geq 1 / 4$; reação de imunofluorescência indireta $\geq 1 / 40$; ELISA reagente) para infeção por T.cruzi, atendidos no ambulatório do GEDoCh/HC FCM/Unicamp, de 1975 a 1990. No presente estudo tomouse em consideração a faixa etária, sexo, raça (negróide ou caucasóide), o diagnóstico de HA, segundo os critérios da American Heart Association ${ }^{6}$ e a caracterização das formas clínicas da moléstia de Chagas, em: forma clínica I - indivíduos na forma indeterminada; II - cardiopatas sem cardiomegalia e/ou insuficiência cardíaca; III - pacientes com cardiomegalia e/ou insuficiência cardíaca; IV - portadores de megavísceras ao nível de trato digestivo; V - associação entre megavísceras e alterações cardíacas não caracterizadas como cardiomegalia e/ou insuficiência cardíaca; VI - pacientes com megavísceras, cardiopatia e insuficiência cardíaca e/ou cardiomegalia.
Os dados relativos aos chagásicos hipertensos foram confrontados com os obtidos na avaliação de chagásicos não hipertensos, excluindo-se nos dois grupos, portadores de outras doenças associadas. Os dados foram analisados e comparados estatisticamente pelo teste do qui-quadrado e os resultados considerados significativamente diferentes para valores de $\mathrm{p} \leq 0,05$.

\section{Resultados}

Nossos achados mostram um maior e significativo ( $\mathrm{p}=0,007)$ comprometimento miocárdico em $56 \%$ dos casos, em vigência da associação da tripanosomíase sul-americana e HA, quando comparados a portadores de doença de Chagas isoladamente (39\% dos pacientes). Também se constatou que as formas mais graves da cardiopatia ocorrem mais freqüentemente em chagásicos hipertensos comparados aos não hipertensos ( $31 \%$ contra $24 \%$ ), embora não se tenha constatado diferença estatisticamente significativa $(p=0,158)($ tab. I).

\begin{tabular}{|c|c|c|c|c|c|c|c|}
\hline Forma clínica & $1 *$ & $2 *$ & 3 & 4 & 5 & $6^{*}$ & Total \\
\hline Chagásicos & 115 & 64 & 61 & 29 & 19 & 39 & 327 \\
\hline Hipertensos $(\mathrm{CH})$ & $35 \%$ & $20 \%$ & $19 \%$ & $9 \%$ & $6 \%$ & $12 \%$ & $37 \%$ \\
\hline Chagásicos não & 281 & 63 & 95 & 49 & 23 & 40 & 551 \\
\hline Hipertensos $(\mathrm{CNH})$ & $51 \%$ & $11 \%$ & $17 \%$ & $9 \%$ & $4 \%$ & $7 \%$ & $63 \%$ \\
\hline
\end{tabular}

\begin{tabular}{|c|c|c|c|c|c|c|c|c|}
\hline \multicolumn{9}{|c|}{ Tabela II - Distribuição dos pacientes chagásicos portadores ou não de hipertensão arterial por faixa etária } \\
\hline $\begin{array}{c}\text { Faixa etária } \\
\text { (anos) }\end{array}$ & $<25^{*}$ & $25-35 *$ & $36-45$ & $46-55^{*}$ & $56-65 *$ & $>65^{*}$ & Desconhecida & Total \\
\hline Chagásicos & 8 & 48 & 90 & 102 & 46 & 15 & 18 & 327 \\
\hline Hipertensos & & & & & & & & \\
\hline$(\mathrm{CH})$ & $2 \%$ & $15 \%$ & $28 \%$ & $31 \%$ & $14 \%$ & $5 \%$ & $6 \%$ & $37 \%$ \\
\hline $\begin{array}{l}\text { Chagásicos } \\
\text { não }\end{array}$ & 79 & 159 & 153 & 87 & 43 & 10 & 20 & 551 \\
\hline $\begin{array}{l}\text { Hipertensos } \\
\text { (CNH) }\end{array}$ & $14 \%$ & $29 \%$ & $28 \%$ & $16 \%$ & $8 \%$ & $2 \%$ & $4 \%$ & $63 \%$ \\
\hline
\end{tabular}

\begin{tabular}{|c|c|c|c|c|c|c|c|c|c|}
\hline $\begin{array}{l}\text { Raça } \\
\text { Sexo }\end{array}$ & $\begin{array}{l}\mathrm{Ca} \\
\mathrm{Ma}\end{array}$ & & & & & & & & \\
\hline Faixa etária (anos) & $<45$ & $>45$ & $<45$ & $>45$ & $<45$ & $>45$ & $<45$ & $>45$ & Total \\
\hline $\begin{array}{l}\text { Chagásicos } \\
\text { Hipertensos } \\
\text { (CH) }\end{array}$ & $\begin{array}{c}32 \\
18 \%\end{array}$ & $\begin{array}{c}33 \\
18 \%\end{array}$ & $\begin{array}{c}23 \\
13 \%\end{array}$ & $\begin{array}{c}57 \\
32 \%\end{array}$ & $\begin{array}{c}6 \\
3 \%\end{array}$ & $\begin{array}{c}5 \\
2 \%\end{array}$ & $\begin{array}{c}12 \\
7 \%\end{array}$ & $\begin{array}{c}11 \\
7 \%\end{array}$ & $\begin{array}{r}179 \\
36 \%\end{array}$ \\
\hline $\begin{array}{l}\text { Chagásicos } \\
\text { não } \\
\text { Hipertensos } \\
\text { (CNH) }\end{array}$ & $\begin{array}{c}130 \\
0 \%\end{array}$ & $\begin{array}{c}52 \\
16 \%\end{array}$ & $\begin{array}{c}61 \\
19 \%\end{array}$ & $\begin{array}{c}32 \\
10 \%\end{array}$ & $\begin{array}{l}27 \\
8 \%\end{array}$ & $\begin{array}{c}7 \\
2 \%\end{array}$ & $\begin{array}{l}10 \\
3 \%\end{array}$ & $\begin{array}{c}5 \\
2 \%\end{array}$ & $\begin{array}{l}324 \\
64 \%\end{array}$ \\
\hline
\end{tabular}




\begin{tabular}{|lccc|}
\hline \multicolumn{4}{|c|}{ Tabela IV - Associação da doença de Chagas e hipertensão arterial em } \\
chagásicos das raças negra e branca
\end{tabular}

Além desses dados, a tabela II mostra uma elevada prevalência da associação entre doença de Chagas e HA nas faixas etárias mais altas: 50\% dos chagásicos hipertensos têm idade $>45$ anos, contra $26 \%$ dos chagásicos não hipertensos. Apenas $2 \%$ dos chagásicos hipertensos têm menos de 25 anos, contra $14 \%$ dos não hipertensos $(\mathrm{p}=0,000)$.

A tabela III mostra que, considerando a presença de elevadas cifras tensionais concomitante à infecção pelo T.cruzi, relacionada à raça, sexo e faixa etária, evidenciou-se uma diferença estatisticamente significativa entre caucasóides do sexo masculino $(\mathrm{p}=0,002)$ e feminino $(\mathrm{p}=0,000)$ e negróides do sexo masculino ( $\mathrm{p}=0,000$ ), distribuídos segundo a idade até 45 anos, ou superior a esta. Porém, entre as mulheres negras, a idade não representou fator discriminante à maior ocorrência de $\mathrm{HA}(\mathrm{p}=0,583)$.

Avaliando-se isoladamente apenas o fator raça, entre os chagásicos caucasóides 35\% são hipertensos. Porém, entre os negróides, esse valor eleva-se para $41 \%$. Entretanto, como mostra a tabela IV, não há diferença significativa entre as raças quando associadas ou não à $\mathrm{HA}(\mathrm{p}=0,320)$.

\section{Discussão}

Os achados deste estudo demonstram uma elevada ocorrência de HA, entre os portadores de infecção pelo T.cruzi, tanto para os negróides como para os caucasóides, já verificado em outros levantamentos ${ }^{4,5}$.

No que se refere à forma clínica, os elevados níveis pressóricos acompanharam-se de maior dano cardíaco, conforme dados da literatura ${ }^{5}$. Enquanto $51 \%$ dos chagásicos não hipertensos avaliados nesta amostra foram classificados na forma indeterminada, 57\% dos chagásicos hipertensos tinham algum grau de comprometimento cardíaco.

Outra importante observação do presente estudo confirma dados anteriores quando demonstra que entre os chagásicos hipertensos, 50\% situavam-se acima dos 45 anos comparados à incidência de $26 \%$ entre o grupo de chagásicos não hipertensos. Estas observações demonstram que a elevação pressórica está associada às faixas etárias $>45$ anos, já verificado na população não portadora de doença de Chagas ${ }^{7,8}$. Nosso estudo mostra também que a associação destas duas doenças parece contribuir para o incremento do dano cardíaco, inclusive em termos de descompensação hemodinâmica (presente em $31 \%$ dos chagásicos hipertensos e em $24 \%$ dos não hipertensos). A concomitância de doença de Chagas e HA primária ocorreu em $37 \%$ dos pacientes avaliados, o que indica uma maior morbidade na esfera cardiovascular para uma porcentagem considerável dos portadores de infecção pelo T.cruzi. Adicionalmente, à análise destes dados pode-se presumir que a incidência simultânea destas enfermidades em jovens, pode acarretar uma deterioração funcional do sistema cardiovascular, justamente na fase mais produtiva da vida ${ }^{3,5,9,10}$.

Quando se consideraram os pacientes estudados segundo os fatores raça, sexo e idade, verificou-se que a maior concentração de cifras pressóricas aumentadas ocorreu entre as mulheres brancas $>45$ anos (32\% do total de chagásicos portadores de HA). Este dado avaliado isoladamente poderia traduzir apenas uma característica da população considerada, em relação ao gênero e ao encaminhamento a um serviço de referência ${ }^{11}$.

Ao se avaliar a ocorrência de HA nos sexos masculino e feminino, tanto em chagásicos de raça branca como negra, verificou-se uma significativa predominância da mesma entre as mulheres (46\% das 173 chagásicas caucasóides eram hipertensas contra $26 \%$ dos 247 chagásicos da mesma raça, assim como $61 \%$ das 38 chagásicas negróides contra $24 \%$ dos 45 pacientes chagásicos negróides). Outros estudos têm apontado maior ocorrência de níveis tensionais aumentados no sexo masculino ${ }^{7}$. Um levantamento realizado nos Estados Unidos e concluído na década passada, evidenciou uma prevalência de $33 \%$ de hipertensos no sexo masculino e de $27 \%$ no sexo feminino ${ }^{12}$, o que torna intrigante o resultado obtido no presente trabalho, quanto à associação de doença de Chagas e HA no sexo feminino. Mas, é importante lembrar que na população estudada havia um predomínio de mulheres na faixa etária mais elevada $(50 \%$ com idade $\geq 45$ anos, sendo $51 \%$ na raça branca e $42 \%$ na raça negra). No sexo masculino, $33 \%$ tinham idade $\geq 45$ anos (34\% entre os caucasóides e $27 \%$ entre os negróides). Portanto, o fator idade pode estar interferindo nos achados deste estudo em relação à população feminina.

A maior ocorrência de níveis pressóricos elevados em mulheres com mais de 45 anos, poderia ser justificada pela combinação de ganho de peso e alterações hormonais próprias do período pós-menopausa ${ }^{7}$. Entretanto, entre as pacientes chagásicas negróides, estas características não ficaram evidentemente claras, já que não houve diferença segundo a idade. É possível que nesse grupo a herança genética, a ingestão de sal e de estresse, entre outros fatores, tenham uma importância considerável, independentemente da faixa etária ${ }^{7}$.

Verificou-se, portanto, que a maior ocorrência de HA na população avaliada neste estudo, associa-se ao sexo feminino. Deve-se levar em conta a possibilidade de que fatores de ordem socioeconômica, tais como menores níveis de escolaridade, qualificação profissional e renda estejam contribuindo para este achado ${ }^{11,13}$. Esta condição pode ser melhor evidenciada nas pacientes chagásicas do sexo feminino do que no sexo masculino ${ }^{11}$.

Por outro lado, não se pode desconsiderar a disautonomia presente nos pacientes chagásicos ${ }^{1,2,14} \mathrm{e}$ sua eventual repercussão no comportamento das cifras pressóricas. 
Estudos funcionais têm demonstrado uma nítida depressão da atividade parassimpática nesta doença ${ }^{2,15}$. Entretanto, também tem sido demonstrada uma resposta adrenérgica deficitária nos indivíduos infectados pelo T.cruzi ${ }^{1,16}$. Além disso, já se evidenciou uma resposta autonômica simpática diminuída em chagásicos hipertensos, à semelhança do que se verifica em não hipertensos ${ }^{17}$. Este padrão distinto de resposta pode ser atribuído à diversidade de cepas do mesmo parasita ou a diferentes estágios da disautonomia ${ }^{5,18,19}$.

Além disso, é bastante provável que outros fatores, inclusive aqueles relacionados à excreção renal de sódio, à função do sistema renina-angiotensina-aldosterona e a defeitos moleculares no sistema de transporte de membrana celular ${ }^{5,720,21}$, possam estar relacionados mais diretamente à associação entre HA e doença de Chagas.

Finalmente, quando no presente estudo os pacientes são avaliados por sua distribuição racial, evidencia-se uma nítida elevação pressórica nos indivíduos negróides (41\% de hipertensos negróides contra $35 \%$ verificada na raça branca), embora sem significância estatística. Não se pode desconsiderar, entretanto, que os indivíduos de raça negra constituem apenas $17 \%$ da população estudada. Por outro lado, a literatura especializada tem documentado a maior ocorrência de HA em negróides, independentemente de outras doenças associadas ${ }^{7-9}$.

Em resumo, os achados deste estudo sugerem uma possível interação prognóstica na evolução do comprometimento cardiológico entre duas afecções altamente prevalentes em nosso meio. Futuros ensaios devem ser implementados na tentativa de entender a combinação dos múltiplos fatores que possam estar participando deste intrincado quadro fisiopatológico.

\section{Referências}

1. Palmero HA, Caeiro TF-El desafio de la enfermedad de Chagas. Medicina 1982; 42: 69-75.

2. Gallo Jr L, Morelo $F^{\circ}$ J, Maciel BC, Marin-Neto JA, Barreto LEM, Lima F ${ }^{\circ}$ EC Functional evaluation of sympathetic and parasympathetic system in Chagas' disease using dynamic exercise. Cardiovasc Res 1987; 21: 922-7.

3. Soato GG, Vichi FL, Neto AR, Macedo RR, Carvalho DS - Prevalência de hipertensão arterial em grupos de pacientes chagásicos e não chagásicos em população de hospital geral. Rev Paul Med 1974; 84: 121-3.

4. Medrado Faria MA, Yasuda MAS, Araujo MJO, Lancarotte I, Capano EA, Ruiz Neto PP - Formas clínicas da doença de Chagas na Grande São Paulo. Arq Bras Cardiol 1982; 38: 99-109.

5. Guariento ME, Ramos MC,, Gontijo JAR, Carvalhal SS - Doença de Chagas e hipertensão arterial primária. Arq Bras Cardiol 1993; 60: 71-5.

6. FrölichED, Grim C, Labarthe DR, Maxwell MH, PerloffD, Heidman WH-Recommendations for human blood pressure determination by sphygmomanometers. Hypertension 1988; 11: 209A-22A

7. Oparil S - Arterial hypertension. In: Wyngaarden JB, Smith LH, Bennett JC, ed - Cecil Textbook of Medicine. $19^{\text {th }}$ ed. Philadelphia: WB Saunders 1992; 253-69.

8. Fuchs FD, Moreira LB, Moraes RS, Bredemeier M, Cardozo SC-Prevalência de hipertensão arterial sistêmica e fatores associados na região urbana de Porto Alegre. Estudo de base populacional. Arq Bras Cardiol 1995; 63: 473-9.

9. Debert-Ribeiro MB, Ribeiro AB, Stabile C, Ramos OL - Hypertension and economic activities in São Paulo, Brazil. Hypertension 1981; 3(suppl 2): II233-7.

10. Mendivil GT, Schenone E, Princich J - La pression arterial en jovenes de 18 años de un area endérmica para la enfermedad de Chagas. Medicina 1978; 38 : 741-3.

11. Guariento ME, Parma MF, Orosz JEB, Freitas AG, Morano FG, Rocha RH -
Caracterização da doença de Chagas quanto a gênero. Rev Soc Bras Med Trop 1994; 27(supl 2): 141-1.

12. The Joint National Committee on Detection, Evaluation and Treatment of High Blood Pressure: The 1988 report of the Joint National Committee on Detection, Evaluation, and Treatment of High Blood Pressure. Detailed recommendations for the diagnosis and pharmacologic and nonpharmacologic treatment of systemic hypertension. Arch Intern Med 1988; 148: 1023-38.

13. Guariento ME - Implicações ocupacionais e trabalhistas da doença de Chagas nos centros urbanos. Rev Soc Bras Med Trop 1994; 27(supl 4): 139-41.

14. Koeberle F- The causation and importance of nervous lesions in American trypanosomiasis. Bull WHO 1970; 4: 7-39.

15. Manço JC, Gallo Jr L, Marin-Neto JA, Terra F J, Maciel BC, Amorim DS Alteraç̃os funcionais do sistema nervoso autônomo. In: Cançado JR, Chuster M, eds - Cardiopatia Chagásica. Belo Horizonte: Fundação Carlos Chagas, 1985: 91-8.

16. Guariento ME, Muscelli EO, Gontijo JAR - Chronotropic and blood pressure response to oral glucose load in Chagas' disease. São Paulo Medical Journal/ RPM 1994; 112: 602-6.

17. Guariento ME, Teixeira CO, Teixeira MAD-Resposta cronotrópica e pressórica em chagásicos hipertensos submetidos à ingestão oral de glicose. Rev Soc Bras Med Trop 1994; 27(supl 2): 143.

18. Cannon WB - A law of denervation. Am J Sci 1939; 198: 737-50.

19. Caeiro TF, Palmero HA, Iosa DJ - Estudio del reflejo barorreceptor en la enfermedad de Chagas. Medicina 1989; 40: 27-32

20. Acquatella H, Puigbó JJ - Renal hemodynamics and sodium excretion after saline infusion in patients with Chagas' disease. Arq Bras Cardiol 1974; 27: 551-62.

21. Diament J, Forti N, Gianinni SD - Alterações metabólicas na doença de Chagas. Arq Bras Cardiol 1981; 37: 189-94. 